\title{
Shared instrumentation facilities: Benefiting researchers and universities, and sustaining research excellence
}

\author{
By T. Amanda Strom, Greg Haugstad, Jonathan Shu, and Ram Seshadri
}

\section{The case for shared experimental facilities}

Consider a hypothetical scenario: A new faculty member is happy to start her independent university faculty career with a substantial startup package, which allows her to purchase a state-of-the art diffractometer. Research is progressing well, and the group is productive. However, come year four, problems commence. The original service contract for the diffractometer has expired, and renewing it is prohibitively expensive for the laboratory. The $\mathrm{x}$-ray tube is reaching the end of its life, and despite the fact that several other groups are also using the diffractometer, no agreement is in place to contribute maintenance funds. The diffraction expert in the laboratory has left for the next stage of his career, and instrumentation upkeep and training of new users are suffering. What is the faculty member to do?
Here is an alternative scenario: A university faculty candidate, during a second round of visits in the hiring process, is somewhat surprised to learn that she will not have her own diffractometer, but instead, the university will purchase a new one that will be housed in a shared facility. The shared facility model is distinct from a traditional single principal investigator (PI) model in that it is a well-defined space with the necessary infrastructure, populated by research instrumentation serving many investigators, potentially including researchers from outside the university. In the shared facility model, equipment is overseen by highly trained full-time staff members dedicating themselves to training and aiding users and maintaining and communicating the full capabilities of the laboratory. Additionally, the expectation is that everyone, including

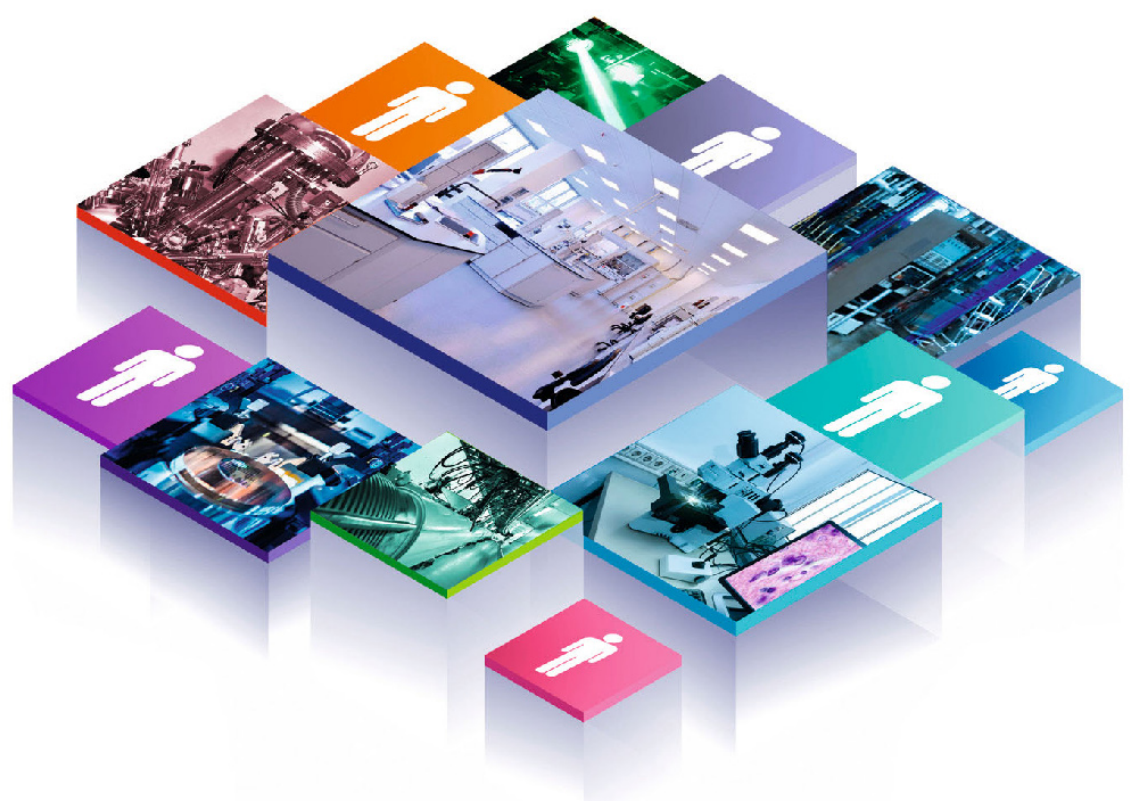

the prospective PI, will pay a usage fee for instrument time that will go toward upkeep, and just one PI or group will not have priority on use.

In effect, this article is about why the faculty candidate in the second scenario should be convinced that the suggested path - notably of relying on shared facilities - can be more beneficial in the long term: to the PI, to the university, and to the research community at large. Several documents and articles have appeared over the years that are relevant to this discourse, including two US National Academy reports $^{1,2}$ and some recent articles. ${ }^{3-5}$

The opinions, examples, and best practices provided in this article were gleaned from participants who met in March 2018 under the umbrella of a US National Science Foundation (NSF)sponsored Shared Facilities Operations Workshop. The workshop participants included faculty and facilities managers from Materials Research Science and Engineering Centers (MRSECs), Materials Innovation Platform (MIP), and National Nanotechnology Coordinated Infrastructure (NNCI) facilities from across the United States. While data management was discussed at length during these meetings and is a central concern for shared facilities, it is not discussed in this article to keep the scope manageable.

\section{Benefits of the shared facilities model}

While there may be rare situations where shared instrumentation is not ideal (e.g., high single-user demand), in the majority of cases, large and diverse bodies of researchers are involved, and shared facilities can

T. Amanda Strom, Materials Research Laboratory, University of California, Santa Barbara, CA, strom@ucsb.edu; Greg Haugstad, Characterization Facility, University of Minnesota, Minneapolis, MN, haugs001@umn.edu; Jonathan Shu, Cornell Center for Materials Research, Cornell University, Ithaca, NY, jbs24@cornell.edu; Ram Seshadri, Materials Research Laboratory, University of California, Santa Barbara, CA, ramseshadri@ucsb.edu 
provide wide-ranging benefits. The impact of shared facilities is felt at many levels, from individual researchers, including staff, to a campus-wide level, and finally to the research community as a whole.

Universities benefit from shared facilities through the visibility of well-defined and accessible instrumentation, which, in turn, aids in faculty recruitment and retention. Interested faculty have input on equipment acquisitions, redundant purchases can be avoided, and equipment usage is maximized. In comparison to individual faculty instrumentation, shared facility operations have numerous financial benefits to a university campus that can minimize expenses and increase revenue. Management of short- and longterm expenditures becomes easier with the stability of a large pool of instruments and personnel. The ability to spread costs invaluable. In addition to financial gains, as a facility's user base grows, their communications become more impactful as they reach a broader audience. Vendors are more likely to participate in collaborative events, such as workshops and short courses, that reach a large and diverse research audience. Institutions are also able to build and leverage long-term relationships with vendors to access not only competitive pricing on equipment over a wide range of instrumentation is and maintenance but also improved service. In the collective experience of the authors, there have been several instances of equipment vendors significantly reducing prices and providing other benefits because of their awareness of the facility user base. A less tangible benefit of shared facilities to the university is that they serve as hubs for facilitating communication between research groups, and become a magnet for industrial collaborations as well as employment opportunities. In the three universities the authors are affiliated with, they are aware of numerous examples of collaborations that originated through conversations in shared facilities.

Considering the many benefits in highquality shared facilities, there is considerable anecdotal evidence of their role in attracting competitive graduate students and postdoctoral fellows, as well as new faculty members, to an institution. In turn, researchers in shared facilities improve their knowledge of analytical science, thus making them more competitive technically for both the initial job market and in later careers. This point holds true not only for hands-on users but also for those who seek analytical services and collaboration with facility staff. Future careers benefit from both an intimate understanding of the operational principles of instrumentation (from hands-on immersion) and from exposure to advanced and custom capabilities, whether developed in the course of usage or in collaboration with experts.

For the individual researcher, whether a faculty member or a student, shared facilities reduce the burden and distraction of installing and then sustaining tools that are critical for research progress. Access to expert staff saves time for the faculty member and students and provides consistent and effective training with a depth of knowledge and expertise not often achieved through peer-to-peer training. Shared facilities provide opportunities for staff to learn new techniques and increase knowledge and effectiveness through engagement with researchers as well as teamwork with other technical staff. A team of dedicated technical staff, able to assist each other, makes for more efficiently managed shared facilities as well as better overall service for researchers. Facility staffing impacts research by reducing the reliance upon student-tostudent knowledge transfer and by providing expert advice or outright contributions through advanced data acquisition and analysis strategies. Additionally, shared facilities provide visibility and access to all available capabilities within the facility, and help to foster a community of interdisciplinary users who can cross-pollinate research ideas as well as strategies for new modes of facilities usage.

Shared facilities, when
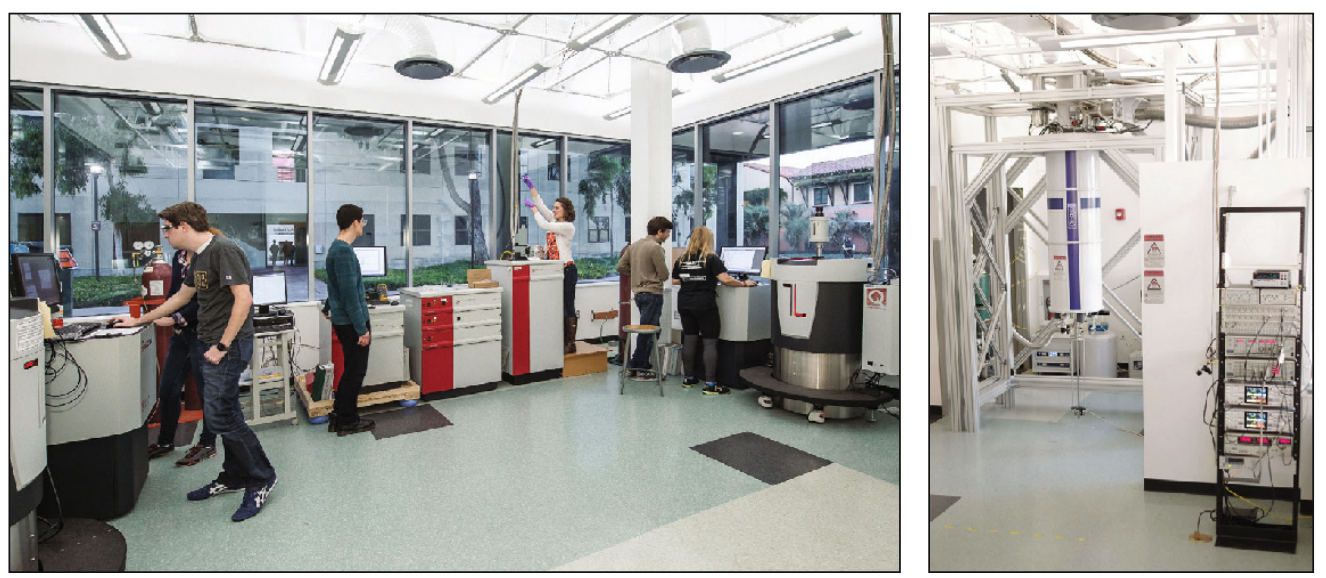

Figure 1. Low-temperature facility at the Materials Research Laboratory (a US National Science Foundation Materials Research Science and Engineering Center [NSF-MRSEC]) at the University of California, Santa Barbara. (Left) Closed-loop cryogenic systems for electrical, thermal, and magnetic property measurements. Two of the instruments were purchased with NSF-MRSEC funds, while the third was an acquisition made from startup funds to an individual investigator. (Right) A closed-loop milli-Kelvin refrigerator with a 14-T magnet in the same facility, acquired through an NSF Major Research Instrumentation project. appropriately managed, are also effective in supporting local industry at all stages of growth. The presence of accessible shared facilities in a geographic area benefits the community not only by enabling and strengthening a startup culture, but also by supporting midto large-sized companies that benefit from soughtafter capabilities or expertise at the academic institution. The symbiotic relationship that develops between university 
researchers and industry, including startups, continues to expand the career paths for a skilled workforce.

\section{Components of an effective shared facility}

\section{Space and instrumentation}

Having outlined some benefits of shared facilities, it is worthwhile to explore what makes a successful shared facility. One necessary component is dedicated space, unambiguously assigned to the facility and not to individual research groups. Equally, none of the instruments in the facility should be outside of the management policies of the facility; equipment dedicated to individual research groups thus does not belong in shared facilities. It is worth noting that effective facility policy necessitates that even if an investigator donates a piece of equipment to a shared facility, that investigator will still pay for the use of the equipment if the facility operates on a fee-based structure. While this may seem counterintuitive, the shared facility is committing to complete oversight of the instrument for the remaining lifetime of the instrument, covering personnel time, maintenance, and other related costs. Indeed, institutional compliance offices audit these operational costs, given that they factor into the expenses charged to grants using the instrument. In this regard, reducing the charges for select users, such as donators of equipment, without allocating offsetting funds to compensate for the reductions, is a violation of federal compliance principles, as it transfers the ongoing costs of one federal grant's activities (i.e., costs postdating the equipment purchase) to some other funding source. Ultimately, the cost to the individual investigator is presumably less than if the equipment is not shared, and many other paying researchers benefit by gaining access to the new instrument or capabilities. In fact, shared facilities often rely on this type of investigator support to gain new capabilities or renew existing ones.

When deciding on equipment and tools appropriate for a shared facility, it is important to consider a potentially large and diverse user base that may include researchers from science and engineering, biological/health sciences, and

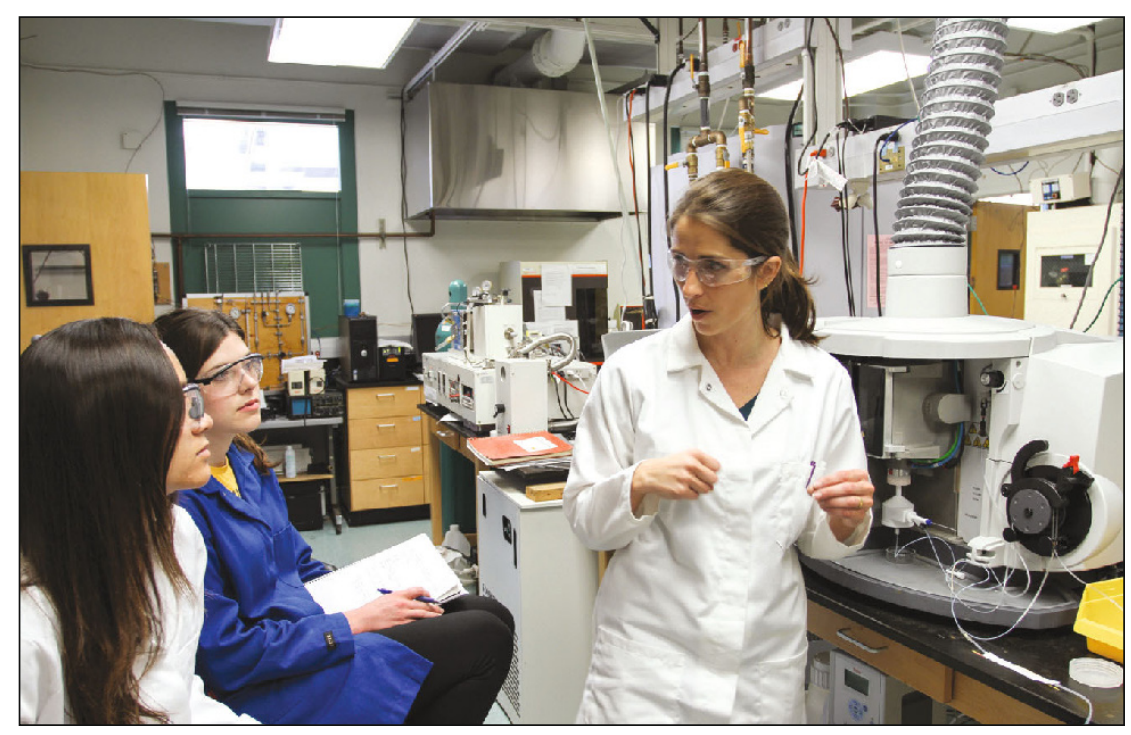

Figure 2. Undergraduate and graduate student researchers at the University of California, Santa Barbara, being trained to use an inductively coupled plasma atomic emission spectrometer. Such training sessions are a major component of the mission of the facilities managers, contributing to the overall research enterprise, and to the development of a skilled workforce.

other domains. In considering diverse users, facilities must often balance the benefits of workhorse, user-friendly instrumentation that can satisfy the needs of many researchers, with the benefits of cutting-edge resources that may attract groundbreaking new research. To this end, strategic planning in shared facilities can benefit from committee and institutional oversight that considers facilities with respect to faculty hiring, research directions, and long-term planning.

In our varied experiences, there are multiple modes for acquiring instrumentation that run the gamut from donations to investigator startup funds, other university sources (e.g., internal competition or faculty retention), to industry partnerships, to grants from foundations and state and federal entities (Figure 1). In terms of competing for an equipment grant from state and federal entities, shared facilities have an advantage in that many competitive grant processes require the demonstration of effective management policies and a substantial user base, both of which are inherent in a properly managed shared facility. In addition to grants and donations, certain permissible user fees can be used for equipment purchases. User fee income alone is not enough to fully support most shared facilities; instead, substantial operational costs may be supported by the home institution or, in some cases, grant funding.

Cutting-edge instrumentation in dedicated spaces is often under-utilized in the absence of effective management policies, practices, and personnel. Good management implies financially sustainable practices, where everyone has equal access to the equipment and all usage is fee-based, with the possible exception of developmental work that adds to facility capabilities. Active stewardship also includes recognizing and responding to the needs of the user base: renewing, supplementing, and discarding tools and equipment as appropriate.

\section{Staffing}

Technical staff who are knowledgeable, welcoming, and act as mentors/collaborators to researchers is key to a successful shared facility. ${ }^{6}$ Technical staff members generally have a wide range of serviceoriented responsibilities, as they may need to troubleshoot and repair instruments, teach best practices, develop research plans, analyze samples, and interpret results. Technical competence alone (i.e., merely teaching someone how to run an instrument) cannot sufficiently meet the needs of a diverse research 
community with distinct and changing needs. Given the importance of staffing to a successful shared facility, an in-depth discussion of the topic is appropriate.

Staff members act as institutional curators of knowledge in analytical methods and facility capabilities. The shared facility staff must stay abreast of the field through usage, development, and collaboration as well as through the scientific literature and vendor newsletters, and participation in conferences. Networking with the technical staff of other university, government, and industry core facilities is also an invaluable resource. In addition to the less tangible skills, a key role the facility's staff play is in the ability to troubleshoot, diagnose, and potentially fix instrumentation problems, and to communicate pertinent information to service personnel. Often such diagnostics require advanced understanding (e.g., of electronics, detectors/transducers, and computer systems). Expert staff members may also act to expand capabilities through upgrades/enhancements and to advocate for the funding of such expansions.

In support of a facility's staff, institutions need to be proactive in creating and implementing codes of conduct for users regarding appropriate recognition of technical staff in publications consistent with journal or professional society guidelines. These guidelines usually consider the expert contribution of measurement science-whether in experimental design, special data acquisition strategies, or data analytics and interpretation- to warrant co-authorship and demand that the resident expert be accountable to the technical community. If co-authorship is not appropriate, then acknowledgment of a staff member by name (i.e., not the facility as a whole) should be the norm when any contribution goes beyond basic training and routine laboratory stewardship.

Regarding professional development, institutions need to establish rewarding career pathways for facility staff members by encouraging and supporting external visibility through journal publications and conference attendance, for example. All of these activities are synergistic with the needs and goals of a university in generating knowledge and serving the broader community. Finally, competitive

\section{(1) Clearinghouses for Shared Facilities Consortia}

Materials Research Facilities Network: www.mrfn.org

National Nanotechnology Coordinated Infrastructure: www.nnci.net

Synchrotron Light Sources: lightsources.org/lightsources-of-the-world

Neutron Sources: www.ncnr.nist.gov/nsources.htm

Electron Microscopy: www.microscopy.org/resources/laboratories.cfm

Cryomicroscopy: commonfund.nih.gov/cryoem

compensation of staff is typically the largest expense for a facility. However, that expense is justified by the net benefit to research and educational endeavors of the institution, making the employment and retention of a highly skilled staff a more than worthwhile investment.

\section{Training of users}

Despite the fact that it is an intrinsic part of the educational enterprise, an often overlooked aspect of shared facilities is the training of users in the proficient use of instrumentation. In our collective experience, users trained in shared facilities run the gamut of expertise from undergraduate interns to $\mathrm{PhDs}$ with a high degree of domain expertise. The latter may include faculty members and users from industry (Figure 2). ${ }^{7}$ Consequently, facilities staff members involved in such training need to be adaptive in their methods and be aware that meaningful staff-trainee relationships require flexibility. If the trainees can learn from the staff member and vice versa, research can thrive. Engaged users collaborating closely with facility staff can provide feedback leading to the advancement of capabilities through upgrades, new attachments, and novel modes of use in addition to propagating further advances. Besides training users for routine applications, in some cases it is appropriate for staff members to carry out the complete experiment and data analysis. From an educational standpoint, users benefit from exposure to advanced techniques mastered by experts.

An interesting challenge that frequently arises in the course of training novice users is distinguishing between the science of the measurement and the proficient use of instrumentation. Facilities staff members often (and inevitably) step in to fill gaps in the foundational scientific training of the researcher, thereby far exceeding their mandate of training for instrument operation per se. While not an intended role of facility staff, this contribution to the educational enterprise deserves greater recognition than it currently receives. As an example, training users to operate a thermogravimetric analyzer often evolves into the science of sample decomposition versus combustion or reduction. A second example is in training users to operate a magnetometer, which naturally introduces or strongly reinforces learning regarding the units of magnetic measurement, their interconversion, and their interpretation. Continuous learning is key, and therefore user training is rarely a single two-hour session, but an ongoing venture with a researcher.

Finally, training of users by shared facilities staff members contributes greatly to workforce preparedness. In addition to providing users with extra knowledge in analytical science, the training can make them more competitive in the job market and strengthen their future technical career growth. More specifically, training in shared facilities can provide a direct link to employers and employment; in fact, shared facility managers are often a resource for commercial laboratories looking for specific skill sets.

\section{Maximizing the impact of shared facilities}

A successful facility is one that benefits the department or center, the institution, and the community. To that end, there are tools and strategies - utilized at the laboratory, university, and local government 
level - that can maximize the efficiency and impact of shared operations. For example, publicizing the capabilities and expertise embodied within a facility to a range of stakeholders through open houses, outreach, and at meetings and workshops is beneficial advertising. Recruiting faculty advocates of a facility is also a crucial opportunity for facility promotion, but in our experience, user word-of-mouth is one of the best drivers of facility usage. An important corollary is that a productive user is a happy user, and a happy user is the best advertisement for the facility. The quality of training, service, and capabilities therefore influences the long-term sustainability of the facility in ways that may not be immediately obvious.

Concerning increasing efficiency, a number of commercial and open-source tools for facilities management, from training and instrument reservation to billing and expenses, have become widely available in recent years. These tools help reduce the administrative burden on facilities staff, making reservations more systematic (leading to happier users) and, most importantly, increasing operational efficiency overall. Anecdotally, the transition from a paper-based reservation and billing system to a more formal computer-controlled system inevitably results in increased revenue for the facility. More often than not, the increase in revenue compensates the added cost of a facilities management system.

From a broader perspective, universities or centers can employ several strategies to facilitate and support access to shared facilities. Consistent university policies aid in making facilities more available and attractive to users. An example is the centralization of the paperwork required of non-university users or the standardization of safety training in a manner that allows users to access multiple facilities in the university more efficiently. Universities can also lev-erage state resources that promote R\&D. Several state programs in the United States (e.g., New Jersey, Illinois, New York, and Pennsylvania) foster innovation and collaboration between industry and academia. These programs can support university-based industrial outreach groups that act as liaisons and continuous points of contact for commercial innovation projects. Industry partnerships benefit all parties; the companies gain insights, university faculty become engaged, students gain contacts, and these collaborations can lead to shared facility usage, utilizing excess hours that may otherwise be unused.

Finally, national and local databases of shared facilities, including lists of

\section{The Case for Shared Facilities (SFs)}

Investment in SFs results in long-term benefits to the university through the recruitment of talent and to the community as a driver of economic growth by enabling and sustaining a startup culture.

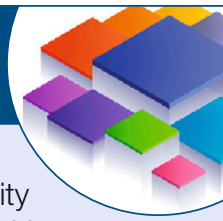
Dedicated space housing both routine and cutting-edge instrumentation and managed by engaged staff, are the fundamental components of a sustainable SF.

Expert and service-oriented staff members are critical for a first-rate SF; they require and warrant the largest investment from stakeholders.

SFs play a significant role in the training and education of the future workforce, and in the process, build a mutually beneficial relationship between the university and industry.

- The impact of SFs can be maximized by investment in instrumentation databases such as MRFN.org, advertising and outreach to the community, and effective university and state policies that support the SFs through funding, contacts, and initiatives.

equipment, services and capabilities, contact details, and policies, can greatly increase the visibility of facilities and help connect users with tools and expertise. One example of an effort in this direction is the US NSF-MRSEC program-supported Materials Research Facilities Network or MRFN (www.mrfn.org) that is a clearinghouse of NSF-supported facilities associated with materials research. Interestingly, the model of the MRFN inspired one of our universities (University of California, Santa Barbara) to create a local database of shared facility equipment. Sidebar (1) lists some key websites associated with shared instrumentation in the United States.

\section{Closing remarks}

Shared facilities at universities can provide a huge number of advantages and efficiencies, some of which are highlighted in Sidebar (2), but the greatest benefit that many of us would attest to is that they enable research to be carried out faster.

\section{Acknowledgments}

We gratefully acknowledge the US National Science Foundation (NSF) for supporting the workshop that led to this contribution through NSF DMR 1818957, and the many workshop participants who shared their insights. The authors are solely responsible for the opinions expressed in this article.

\section{References}

1. National Research Council. Midsize Facilities, The Infrastructure for Materials Research (National Academies Press, Washington, DC, 2006).

2. National Academy of Sciences, National Academy of Engineering, and Institute of Medicine. Advanced Research Instrumentation and Facilities (National Academies Press, Washington, DC, 2006).

3. M. Morales, R. Pepperkok, R. Schlapbach, A. Tiran, D. Meder, G. Van Minnebruggen, EMBO Rep. 17, 1088 (2016)

4. P. Hockberger, J. Weiss, A. Rosen, A. Ott, J. Biomol. Tech. 29, 79 (2018).

5. D.A. Colón-Ramos, P. La Riviere, H. Shroff, R. Oldenbourg, Nat. Methods 16, 667 (2019).

6. J. Gould, Nature 519, 495 (2015).

7. P. Hockberger, S. Meyn, C. Nicklin, D. Tabarini, P. Turpen, J. Auger, J. Biomol. Tech. 24, 87 (2013). $\square$

We welcome comments and feedback on this article via email toBulletin@mrs.org. 